\title{
Design and Implementation of a Radar Equipment Training Simulator Based on Training and Maintenance Dual Mode
}

\author{
Zhang zhonghua ${ }^{1, a}$ \\ ${ }^{1}$ Army Engineering College Ordnance Officer School, Hubei Province, China \\ aipenny.ok@163.com
}

Keywords: Simulation training, Operation training and fault repair dual mode.

\begin{abstract}
In order to meet the requirements of the new radar conditioning training, the development of a certain type of radar simulation training device is mainly to replace the radar's actual installation operation process with simulation operations, and to improve the students' operating ability. This simulator is based on Oracle8.1.7 database and Visual C++6.0 development, can achieve radar routine operation, typical fault maintenance training, multimedia teaching, simulation of air targets and training assessment[1]. This paper focuses on the hardware components and control flow of the simulator, and the specific implementation of the software. The three main training modes of the simulator are introduced systematically.
\end{abstract}

\section{Introduction}

Radar Equipment is an Important Information Support Force in Modern Warfare, With automation, complexity, integration, high digital characteristics[2]; At the same time, it involves many disciplines and technical fields, integrating microwave, automatic control, computer applications, optics, power supply and other technologies. Based on this, the quality requirements for operators and maintenance support personnel are higher, This puts forward higher requirements for the training of institutions and the construction of digital teaching resources.

\section{Hardware composition and main functions}

The training simulator is mainly composed of a director platform and a terminal simulation platform, As shown in Figure 1. Internal LAN consists of network communication devices in the simulator, Information exchange based on IP/UDP protocol. The information processor can intervene in the intelligence command system, and the director control platform can use the pre-reserved network interface and RS-232/RS-422 interface to complete the networking with other simulation training equipment and complete the equipment simulation training.

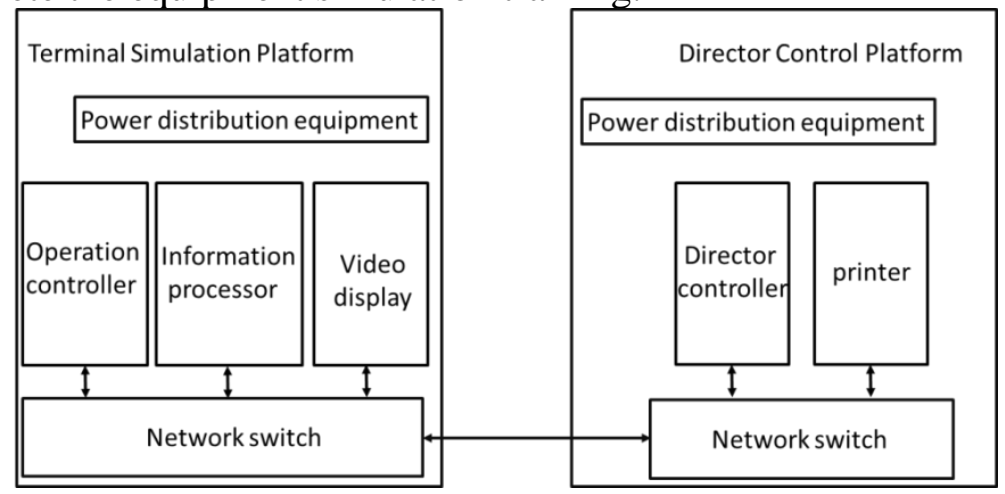

Figure 1 The composition of the simulation training system

\subsection{Director Control Platform}

The director control platform can monitor and control the operation and training conditions of the simulation operation platform under the network environment, set the radar simulation target parameters, and realize the functions of training course setting, target initial parameter setting, 
training situation monitoring and recording, training performance evaluation, data management and network management[3].

\subsection{Terminal Simulation Platform}

Terminal simulation platform and radar equipment have the same structure, Mainly consists of radar operation controller, information processor, video display device, supporting external equipment and shelves; simulation training is mainly completed in the operation control machine and information processor, Operational actions, operational strength, operational response, and operational experience are exactly the same as those of radar equipment, and the operational effects are transmitted back to the director's control platform via the Internet in a timely manner. Video display machine installed system status indication and fault maintenance training software, launch and receive car simulation software and multimedia teaching software[4]. System status indication and fault maintenance training software is mainly used to complete the radar system status and fault information display, guide students to complete the maintenance; Transmitting and receiving car simulation software is mainly used for sub-system simulation and target simulation, providing the display terminal with consistent operation response; Multimedia teaching software adopts a combination of three-dimensional animation, electronic manual and video.

\section{Software design}

The director controller performs task assignment through LAN and database technology, and records the training effect of the operator. Director controller and display analog terminal platform adopt programming interface based on UDP/IP protocol. The network communication process is shown in Figure 2.

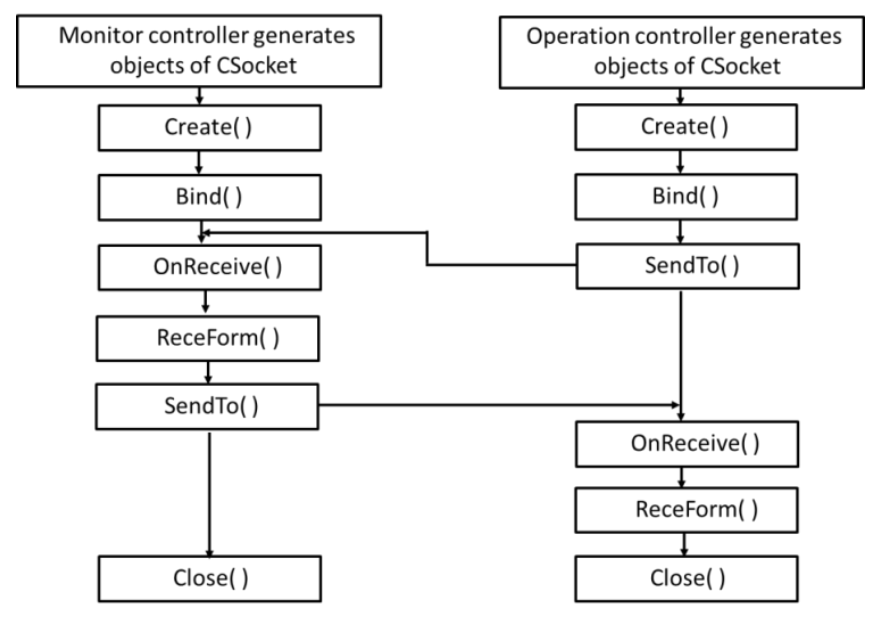

Figure 2 Director Control Platform and Operation Console Communication Process

\section{Operating mode}

\subsection{Target Simulation Mode}

According to the target initial parameters issued by the director controller, the target flight path is simulated after receiving the operation mode control instruction of the terminal simulation platform. The ballistic type targets are modeled according to the ballistic equation, and the flying targets can be set as three kinds of running trajectories in any direction, any position of circumference, sine, and straight line according to needs. The number, distance, azimuth and pitch data of the target generated in real time are sent to the terminal simulator to complete 8 batches of target reconnaissance, surveillance or proofreading of 6 batches of targets. In the reconnaissance mode, the target simulation of the ascending arc segment can be completed, and the simulation of the descending arc segment can be achieved in the calibration mode. Target simulation process shown in Figure 3. 


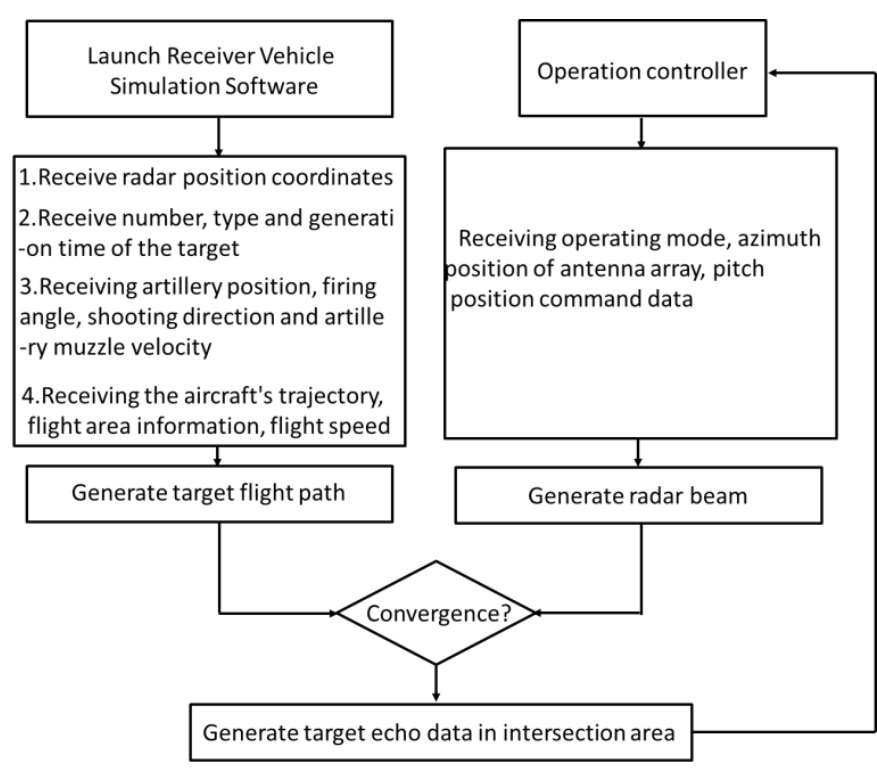

Figure 3 Target Simulation Process

\subsection{Operational training mode}

The director controller first generates the training subjects, training parameters and standard answers, then it sends the training parameters to the launch vehicle simulation software and the terminal simulation platform. The students operate according to the training subjects, and the launch and receive vehicle simulation software simulates according to the control instructions of the terminal simulation platform. The subsystem status response and detection target data are sent to the terminal simulation platform; After training, evaluate training results, get training results and Printout. Operational data information flow chart shown in Figure 4.

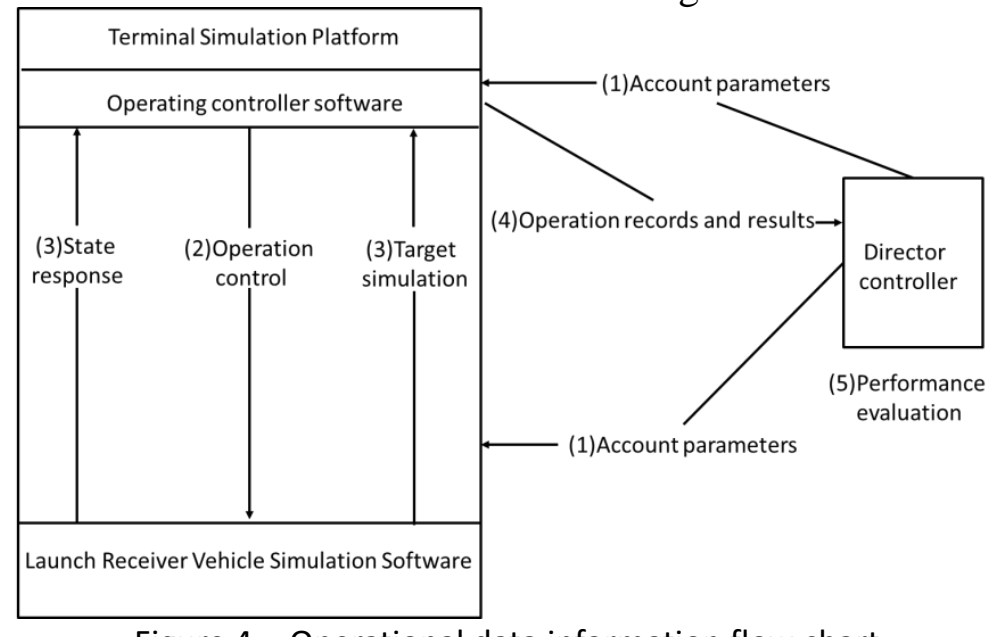

Figure 4 Operational data information flow chart

\subsection{Maintenance Training Mode}

The fault generated by the director controller to transmit and receive vehicle simulation software generates three types of faults: an individual maintenance training fault; a timing fault in the operational training mode; and a random fault in the operational training mode. The transmitter and receiver vehicle simulation software generates fault information and sends it to the operation control terminal. The system status information and fault types are displayed in real time.

\section{References}

[1]Zhang Xuequan. Design and implementation of main control system for meteorological radar simulator[D]. Graduate University of Chinese Academy of Sciences (Chang chun Institute of Optics, Fine Mechanics and Physics), 2005. 
[2] Yang Heyong, Tian Zhaochun, Gao Kai, et al. Simulated Rocket for Artillery Reconnaissance Radar Training: CN, CN 203561295 U[P]. 2014.

[3]ZHANG Yanqing, QU Honggang. Software and Hardware Design of a Radar Target Simulator [J]. Fire Control Radar Technology, 2004, 33(4):61-64.

[4]Tian Jia. Research and Design of Radar Simulator[D]. Dalian Maritime University, 2008. 